\title{
Optimization of Matched and Mismatched Filters in Short Range Pulse Radars using Genetic Algorithm
}

\author{
Hesam Ghaferi \\ Sama technical and vocational training college, Islamic Azad University, Shiraz Branch, Shiraz, Iran \\ E-mail: Hesam.Ghaferi@gmail.com \\ Mohammad Mehdi Pishrow \\ Sama technical and vocational training college, Islamic Azad University, Shiraz Branch, Shiraz, Iran \\ E-mail: mehdipishrow@gmail.com
}

\begin{abstract}
Matched and mismatched filters are considered important parts of a radar signal processing unit. In this paper, we present an approach to optimize the matched filters and mismatched filters in short range pulse radars. For radar, the matched filter coefficients are the complex conjugates of transmitted code. We used binary phase codes as transmitted pulse. The disadvantage of binary phase codes is having high sidelobe levels in the output of correlation function. Thus, we decided to use optimization algorithms for finding binary phase codes with minimum peak sidelobe levels (MPS). After that, we succeeded in producing mismatched filter coefficients (Mis-co) for each code using floating point genetic algorithm (FGA) and we could generate and test the filter coefficients with maximum peak to sidelobe level ratio (PSR). For testing the filter, we plotted ambiguity function for each set of coefficients and tested the filter with Doppler shift.
\end{abstract}

Index Terms-Matched Filter, Mismatched Filter, Pulse Radar, Minimum Peak Sidelobe, Genetic Algorithm.

\section{INTRODUCTION}

With increase applications of modern radar and ultrasonic systems, there is a constant need for increasingly better accuracy and increasing range resolution, with limited peak power. By using pulse compression technique in radar/sonar systems, these requirements can be met simultaneously.

The application of pulse compression coding in radars is to achieve the signal-to-noise ratio (SNR) benefits of a long pulse along with the range resolution of a short pulse $[1,2]$. Pulse compression is a way to achieve the resolution of the short pulses while keeping within the practical constraints of peak power limitation. The process of modulating the transmitted pulse and then correlating the received signal with the transmitted pulse is known as pulse compression [3, 4].

Binary phase coding is one of the basic types of pulse compression, which encodes the transmitted pulse with information that is compressed (decoded) in the receiver of the radar.

The compressed pulse does not have appropriate sidelobes in range and that is the major disadvantage of compression; and for closely spaced targets, the resolution range is limited. A major problem of pulse compression techniques is weak sidelobes suppression. One of the best techniques for sidelobes suppression is using a mismatched filter in the receiver.

Matched and mismatched filter has an important role in pulse compression technique; therefore, its properties and characteristics are explained briefly. The most unique property of the matched filter is that it produces the maximum achievable instantaneous SNR at its output when a signal plus additive white noise is present at the input [5].

There are three ways to optimize the efficiency of the radar system [6]:

- Optimization of the receiver filter (matched or mismatched filter)

- Optimization of the transmitted signal

- Joint optimization of them

In this paper, we have decided to use the third method.

To do this, we first optimize transmission Codes with an innovative approach. The results are given in table (2). Then, using the floating point genetic algorithm that is shown in table (1), we optimize the received filter coefficients.

The rest of the paper is organized as follows: In Section II, the basic concepts involved in the matched filter and auto-correlation function are introduced. In section III, genetic algorithms are introduced. In section IV, problem formulation is derived. Section V presents simulation results. Finally, in Section VI the conclusions of this paper are presented.

\section{REVIEW OF FUNDAMENTAL CONCEPTS}

First, some basic concepts must be introduced. In this 
section, the basic concepts including theoretical foundations of matched and mismatched filter and autocorrelation function are explained.

\section{A. Matched Filter Theory}

Matched filter is obtained by correlating an unknown signal with a known signal or template to detect the presence of the template in the unknown signal. This is equivalent to convolve the complex conjugated timereversed version of the template with an unknown signal. The matched filter is the optimal linear filter to maximize the signal to noise ratio (SNR) in the presence of additive stochastic noise. Matched filters are commonly used in radar, in which a known signal is sent out, and the reflected signal is examined for common elements of the outgoing signal.

The basic concept of matched filters evolved from the effort to obtain a better theoretical understanding of the factors leading to optimum performance of our system.

The characteristics of matched filters can be designated by either a time response function or a frequency response function, each being related to the other by a Fourier transform operation. In the frequency domain, the matched filter transfer function, $\mathrm{H}(\mathrm{f})$, is the complex conjugate function of the spectrum of the signal that is to be processed in the optimum method. Thus, the transfer function of a matched filter has the form $[7,8]$ :

$$
H(f)=K S^{*}(f) e^{-j 2 \pi f T_{d}}
$$

Where $S(f)$ is the spectrum of the transmitted signal $\mathrm{S}(\mathrm{t}), \mathrm{K}$ is a constant for normalization, and $T_{d}$ is a delay constant required to make the filter physically realizable.

The output of the matched filter is obtained as (2):

$$
y(t)=\int_{0}^{\infty} h(t) s(t-T) d T
$$

Where:

$y(t)=$ Auto-correlation.

$h(t)=$ Compression filter coefficients.

$\mathrm{s}(\mathrm{t})=$ Transmitted pulse compression code.

$\mathrm{T}=$ Correlation delay.

\section{B. Auto-correlation function}

The auto-correlation function (ACF) of a discrete coded waveform can be given as (3):

$$
Y(n)=\sum_{n=0}^{N-1} a_{i} a_{i+|n|}^{*}
$$

Main Lobe (ML) is defined as the absolute maximum value of $\mathrm{ACF}$ (4).

$$
M L=\max (Y(n))
$$

Energy of a sequence and Signal to noise ratio is given by (5)

$$
\begin{gathered}
E=2 \sum_{n=1}^{N-1} Y^{2}(n) \\
S N R=\frac{2 E}{E_{N 0}}
\end{gathered}
$$

Where $\mathrm{E}_{\mathrm{N} 0}$ denoted as input noise power.

The peak sidelobe level (PSL) and the integrated sidelobe level (ISL) are two important parameters for radar performance analysis. A measure of the largest sidelobe as compared with the peak of the compression is known as PSL and a measure of the total power in the sidelobes as compared with the peak of the compression is ISL [9]. For an $\mathrm{N}$ bit code:

$$
\begin{gathered}
P S L=\max _{n \neq 0}(|Y(n)|) \\
I S L=10 \log _{10}\left(\frac{E}{N^{2}}\right)
\end{gathered}
$$

Another parameter that has been a subject of considerable interest is the merit factor (MF), which is closely related to ISL [10].

$$
M F=\frac{N^{2}}{E}
$$

Good sequences are associated with small PSLs and large MFs.

\section{FloAting POINT Genetic Algorithms}

GA [11], are heuristic procedures that are often able to locate near optimal solutions complicated problems. To do this, a GA keeps a set of trial solutions, and forces them to evolve towards an acceptable solution. First, a representation for possible solutions must be developed. Then, starting with an initial random population and employing survival-of-the-fittest and exploiting old knowledge in the gene pool, each generation's ability to solve the problem should improve. This is achieved through a four-step process involving evaluation, reproduction, recombination, and mutation $[12,13]$.

\section{A. Heuristic Crossover}

Crossover in GA is a genetic operator that used to vary the coding of a chromosome or chromosomes from one generation to another one. It is similar to the reproduction and biological crossover, upon which GAs are based. A crossover is a process of producing a child solution from more than one parent solution [14]. 
A linear extrapolation of the two individuals is produced. The direction of the search is determined by using values of the fitness function. It produces two children $I^{A(\text { new })}$ and $I^{B(\text { new })}$ from two parents $I^{A}$ and $I^{B}$. According to the following provided $I^{A}$ is better than $I^{B}$ in terms of fitness [15-17]:

$$
\begin{gathered}
I^{A(\text { new })}=I^{A}+r\left(I^{A}-I^{B}\right) \\
I^{B(\text { new })}=I^{A}
\end{gathered}
$$

Where (r) is a random number between 0 and 1.

$$
\begin{aligned}
\text { Feasibility } & =1, \text { if } a_{i} \leq I_{i}^{A(\text { new })} \leq b_{i} \\
& =0, \text { otherwise }
\end{aligned}
$$

Where $a_{i}$ and $b_{i}$ are lower and upper bounds of each variable in population. If feasibility is equal to zero, then another random value (r) is generated and another solution produced. If after ( $\mathrm{t}$ ) attempts no new solution meeting the constraints is found, the operator gives up and produces no children, i.e., the children become equal to parents and stop [15-17].

\section{B. Mutation}

Mutation is a genetic operator used to maintain genetic diversity from one generation of a population of GA chromosomes to the next. It is similar to biological mutation. Mutation varies one or more gene values in a chromosome from its initial state. In mutation, the solution may change entirely from the previous one. Hence, by using mutation, GA can come to better solutions. Mutation occurs during evolution according to a low mutation probability. If it is set too high, the search will turn into a primitive random search. This probability is user-definable [14].

Table 1. The pseudocode of the GA algorithm

1. Start

2. Define parameters:

2.1. transmission code

2.2. value - PSR

2.3. value $-\mathrm{PSL}$

2.4. The population size

3. Initialize fitness function

4. Randomly generate an initial population of Mis-co individuals within the variable constraint range.

5. Evaluate fitness

6. Selection

7. Crossover

8. Mutation

9. Test fitness function

9.1. Create inverse and conjugate(Mis-co)

9.2. Filter operations using (3)

9.3. If the output value in desired range then go to step

9.4. Else return to step 5.
This is the unique operator responsible for the fine tuning capabilities of the system, so that it can escape from local minima. It randomly changes one variable of a parent. It is defined as follows: for a parent $I^{A}$, if the variable $I_{k}^{A}$ was selected at random for this mutation, the result is:

$$
\bar{I}^{A}=\left(I_{1}^{A}, \ldots, \bar{I}_{k}^{A}, \ldots, I_{k}^{A}\right)
$$

\section{PROBLEM Formulation}

Now, the problem can be written in mathematical format.

It is desired to find an N-bit binary sequences for matched filter whose PSLs or ISLs have the minimum value among all $2 \mathrm{~N}$ existing codes and find $\mathrm{M}$-bit $(\mathrm{M}>\mathrm{N})$ sequences for mismatched filter coefficients whose PSRs have the maximum value and SNR loss has the minimum value.

To find N-bit binary sequences for matched filter whose PSLs or ISLs have the minimum value we used (14) and (15).

$$
\begin{aligned}
\text { cost } & =\min \left(Q_{A(M-F)}+Q_{B(M-F)}\right) \\
& =\min \left(\left(\max _{n \neq 0}(|Y(n)|)\right)+\left(\frac{2 \sum_{n=1}^{N-1} Y^{2}(n)}{N^{2}}\right)\right) \\
\text { fitness } & =\max \left(Q_{C(M-F)}+Q_{D(M-F)}\right) \\
& =\max \left(\left(\frac{\max _{n=0}(|Y(n)|)}{\max _{n \neq 0}(|Y(n)|)}\right)+\left(\frac{N^{2}}{E}\right)\right)
\end{aligned}
$$

To find a mismatched filter with the high PSR value we can use (16).

$$
\text { fitness }=\max \left(Q_{s(\text { Mis_co })}\right)=\max \left(\frac{\max _{n=0}(|Y(n)|)}{\max _{n \neq 0}(|Y(n)|)}\right)
$$

\section{Simulation Results}

The main algorithm to optimize the matched and mismatched filters is shown in Fig 1.

As mentioned before, the output of the matched filter is the auto-correlation function of the input signal. So, a good criterion for choosing bi-phase codes is that their auto-correlation has the sidelobes as minimum as possible. According to the equation (14), (15) and Fig. 1 we search for MPS codes that have minimum values of PSL and ISL. The best codes found, are given in Table 2. 


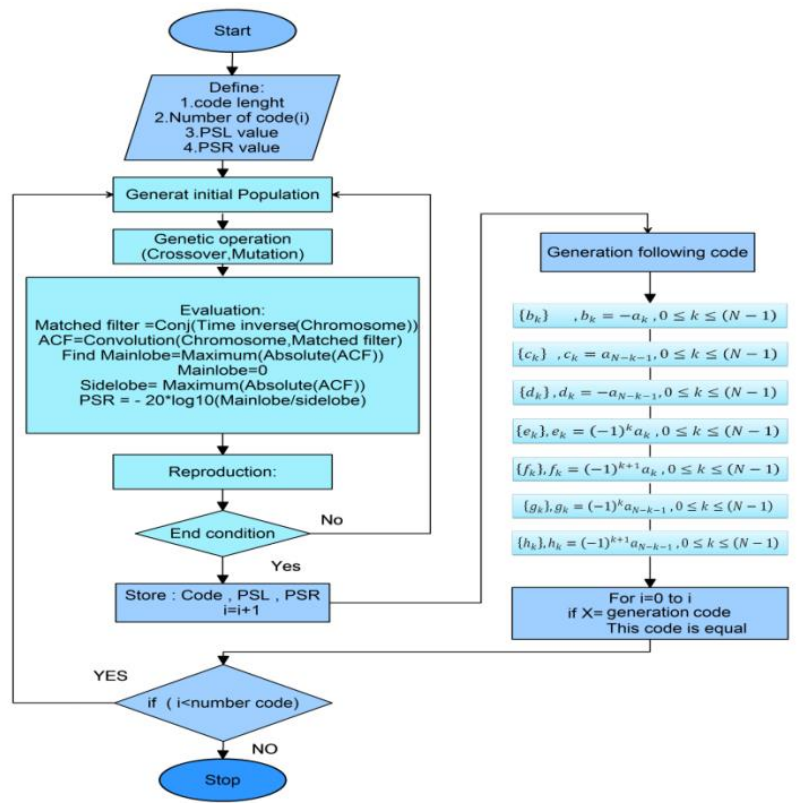

Fig. 1 offers Genetic Algorithm which is an innovative search algorithm used for finding the MPS code with the advantage that the problem of finding equal codes is solved. We used Genetic Algorithm to generate random codes.

The successful extraction of information about the range and velocity is determined by measuring the accuracy of the radar. The performance of the radar system is accurately indicated by the ambiguity function. The response of the matched filter radar receiver to a target displaced in range delay $\mathrm{T}$ and Doppler frequency from a reference target as defined by the ambiguity function.[6] Some of filter ambiguity functions are shown in Fig. 3. Ambiguity function is determined as (17):

$$
|X(T, \varphi)|=\int_{-\infty}^{\infty} \mathrm{u}(\mathrm{t}) \mathrm{u}^{*}(\mathrm{t}+\mathrm{T}) \mathrm{e}^{-\mathrm{j} 2 \pi \varphi \mathrm{t}} \mathrm{dt}
$$

Fig.1. Flow Graph of Innovative Approach using GA

Table 2. Best found BPSK code with Small PSLs and large MFs for 14 to 31code length

\begin{tabular}{|c|c|c|c|c|c|}
\hline Length & PSL & ISL (dB) & PSR (dB) & Code & MF \\
\hline 14 & 2 & -7.1247 & 16.902 & 019FA & 5.1579 \\
\hline 15 & 2 & -6.8942 & 17.501 & 6428 & 4.891 \\
\hline 16 & 2 & -6.6005 & 18.0618 & C2DD & 4.5714 \\
\hline 17 & 2 & -6.5471 & 18.588 & 0B381 & 4.5156 \\
\hline 18 & 2 & -8.1158 & 19.0849 & 2BC26 & 6.48 \\
\hline 19 & 2 & -6.8828 & 19.5545 & 0EEDA & 4.8784 \\
\hline 20 & 2 & -7.2125 & 20 & $5181 B$ & 5.2632 \\
\hline 21 & 3 & -8.1193 & 20.4238 & $9447 C$ & 6.4853 \\
\hline 22 & 3 & -7.9275 & 17.3060 & 38D4DF & 6.2051 \\
\hline 23 & 3 & -7.5033 & 17.6921 & 2AD818 & 5.6277 \\
\hline 24 & 3 & -9.0309 & 18.0618 & 380AD9 & 8 \\
\hline 25 & 2 & -8.5140 & 21.9382 & $7015 B 2$ & 7.1023 \\
\hline 26 & 3 & -8.7570 & 18.7570 & $12540 \mathrm{E} 7$ & 7.5111 \\
\hline 27 & 3 & -9.9350 & 19.0849 & 0F1112D & 9.8514 \\
\hline 28 & 2 & -8.9432 & 22.9226 & 1E2225B & 7.84 \\
\hline 29 & 3 & -8.3137 & 19.7055 & 31FD5B2 & 6.7823 \\
\hline 30 & 3 & -8.8236 & 20 & 3F6D5CE & 7.6271 \\
\hline 31 & 3 & -8.5562 & 20.2848 & 7F1CD95A & 7.1716 \\
\hline
\end{tabular}

Where $u(t)$ is the transmitted envelope waveform, suitably normalized, positive $(\mathrm{t})$ indicates a target beyond the reference delay, and positive $\varphi$ indicates an incoming target.
Next, considering the values of Table 3, the ambiguity function for the optimized code is plotted. 

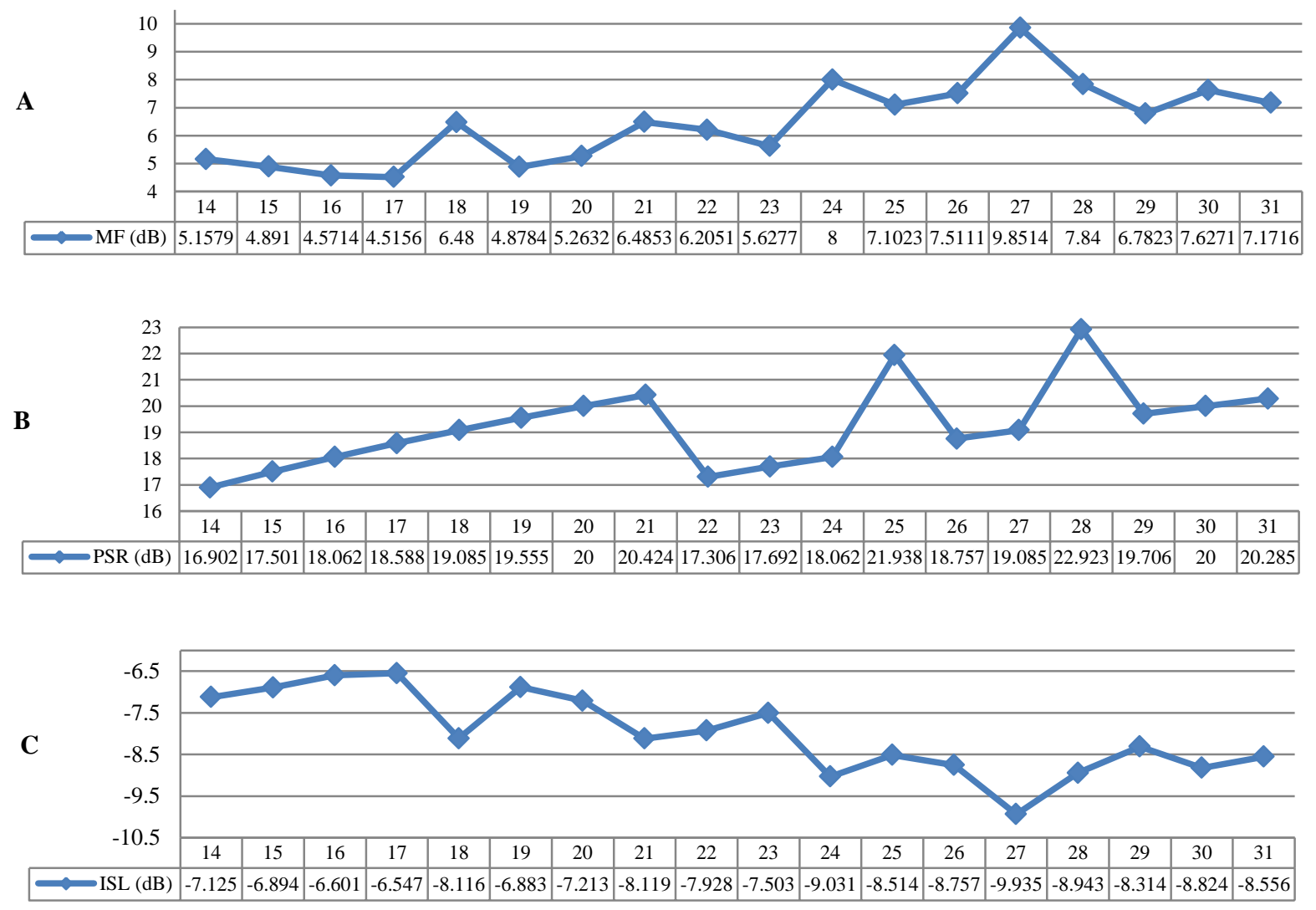

Fig.2. Best Found BPSK Code for 14 to 31code Length Value of (A) MF (B) PSR (C) ISL

Table 3. Parameters to Plot the Ambiguity Function

\begin{tabular}{|c|c|}
\hline Parameters & values \\
\hline Sampling Rate befor M.F & 4 Msps \\
\hline Pulse width & $205 \mu s$ \\
\hline Maximum Velocity & $1000(\mathrm{~m} / \mathrm{s})$ \\
\hline Minimum Velocity & $100(\mathrm{~m} / \mathrm{s})$ \\
\hline Carrier frequency & $780 \mathrm{Mhz}$ \\
\hline Maximum Doppler Frequency & $6.2 \mathrm{KHz}$ \\
\hline Minimum Doppler Frequency & $50 \mathrm{~Hz}$ \\
\hline
\end{tabular}

The value of MF, PSL and ISL for each code is shown in Fig.2.

To increase the peak to sidelob rate of mismatched filter, we used genetic algorithm that its pseudo code is shown in Table 1 and succeeded to generate mismatched filter coefficients, with different lengths and maximum PSR. The optimum mismatched filter coefficients generated for some of the existing codes are shown the Table 2. The PSR values for different filter coefficient lengths are shown in Fig. 4. These generated codes have maximum value of PSR. Some set of generating mismatched filter coefficients are given in Table 4.

To evaluate the performance of binary phase codes existing in Table 2 the ambiguity function was plotted for some of these codes as shown in Fig. 3.

As it is clear from Fig. 3, the found binary phase codes have good performance in presence of Doppler shifts. 

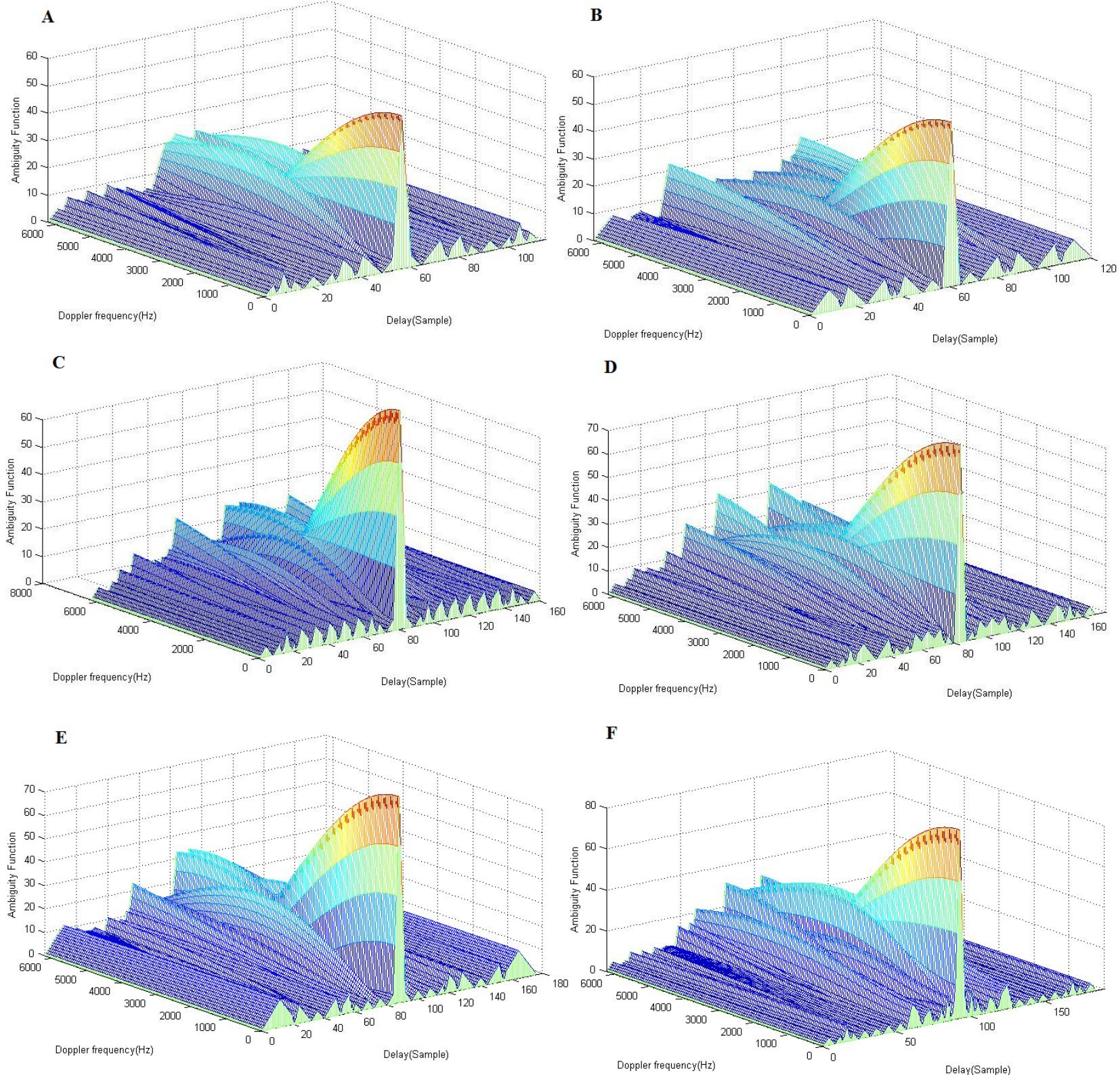

Fig.3. Ambiguity Function of the BPSK code (A) 14-Elements (B) 15-Elements (C) 20-Elements (D) 21-Elements (E) 22-Elements (F) 23-Elements

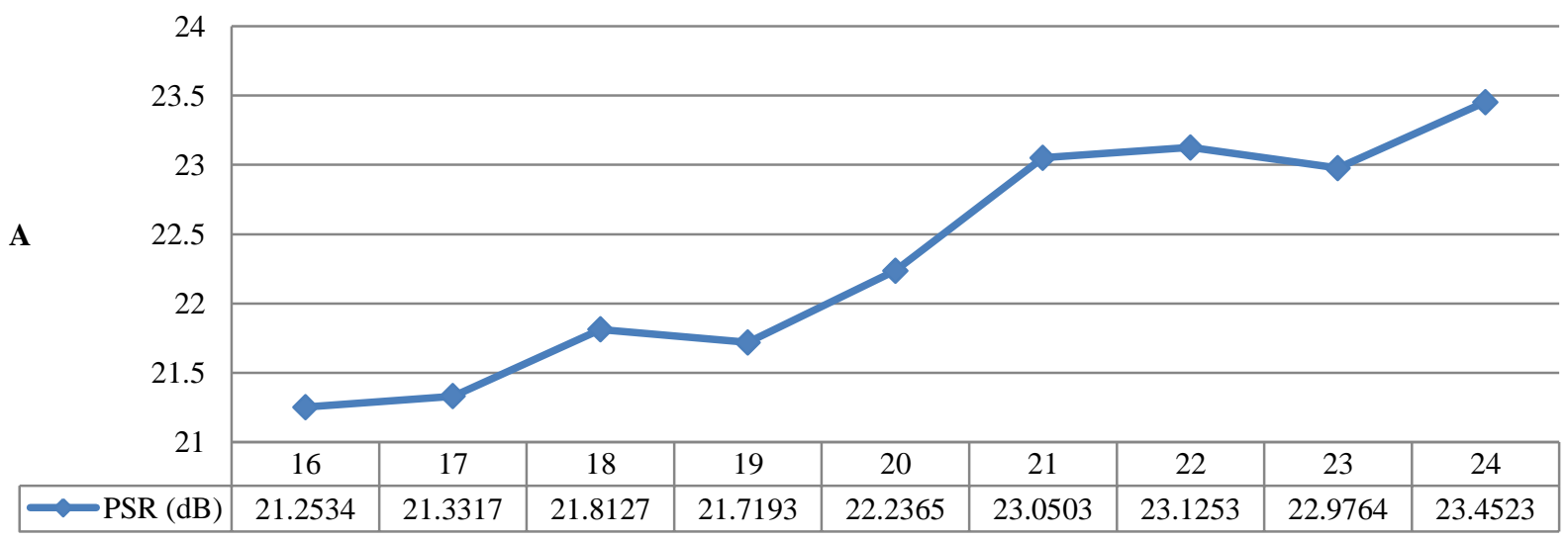



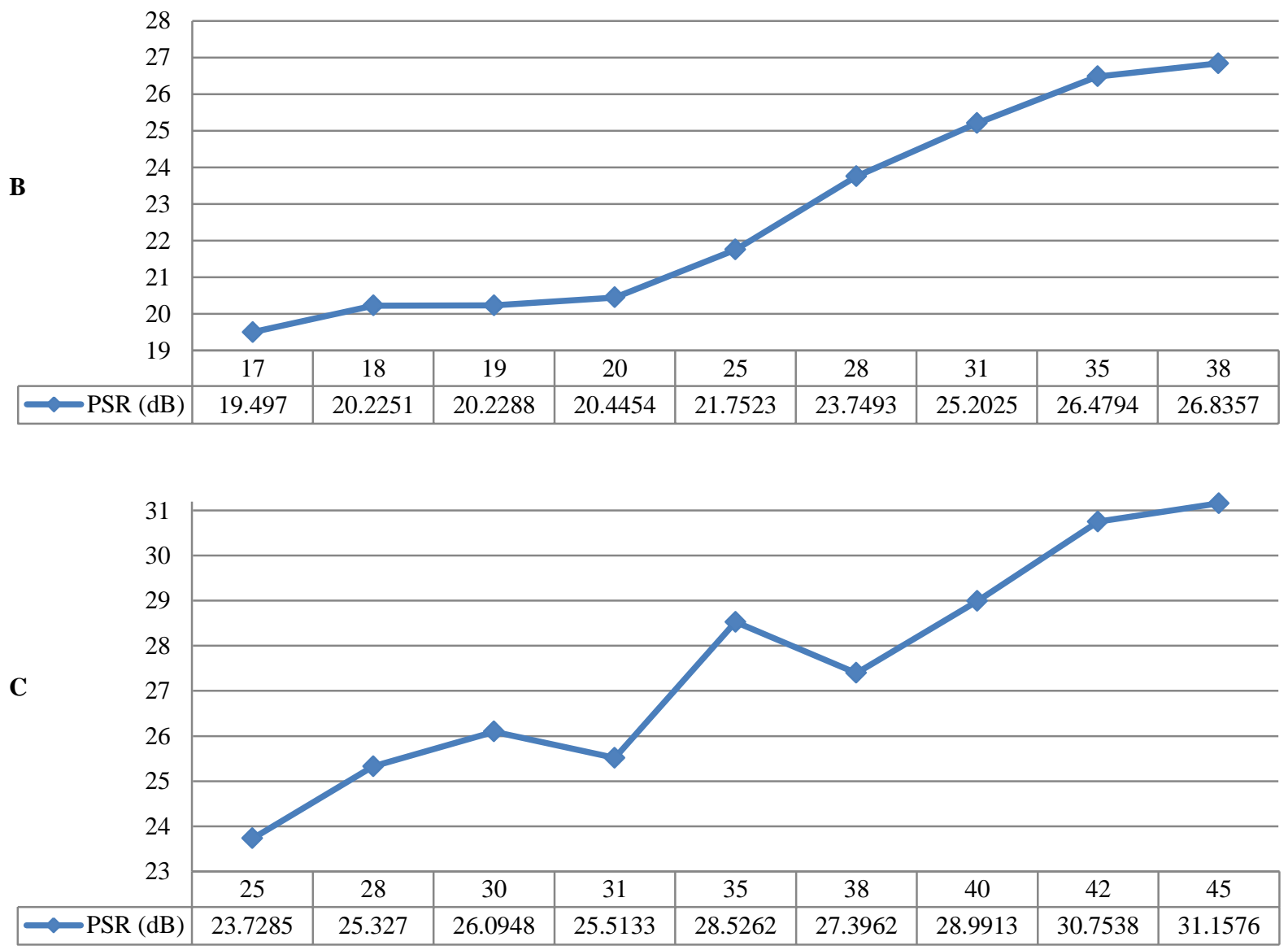

Fig.4. Best Generated Mismatched PSR (A) 14-Elements Transmitted Pulse With Minimum 16 And Maximum 24 Coefficients For Mismatched Filter

(B) 15-Elements Transmitted Pulse With Minimum 17 and maximum 38 coefficients for mismatched filter (C) 21-element transmitted pulse with minimum 25 and Maximum 45 Coefficients For Mismatched Filter

Table 4. 3 of Best Generated Mismatched Coefficients

length

Mismatched filter coefficients

$0.622,0.8582,-0.8682,-0.0529,2.7604,-0.1878,1.0262,-0.5437,1.7051,2.2593,0.7342,4.3455$, -

$3.8429,5.6113,5.1816,-2.4478,6.0784,-2.5792,5.2353,5.4747,4.662,-3.9209,2.3677,6.5386,4.4641$, $3.9132,-2.9193,-2.8904,-3.8566,-2.8541,3.293,3.2979,-0.4137,-0.7456,0.3092,-0.1542,-0.4711,1.1773$, $1.6169,-0.1250,-1.0538,-0.2509$

$15 / 35$

$-0.358,-0.9795,-0.5454,-0.8849,-1.8934,1.1999,2.9322,-1.2431,-0.5967,3.188,0.1583,-1.4407,1.4237$, $-1.2211,-2.185,1.4861,-5.9708,-7.6379,6.6725,4.7676,-5.7129,6.2525,6.1321,3.0117,6.1759,-4.8769$, $1.9926,-3.0696,4.3211,0.9422,2.4236,-0.9082,-0.2956,-0.5084,0.8794$

$14 / 22$ $-0.0369,2.6939,-0.38,-0.9704,-1.6966,-2.2123,4.5541,6.1841,-4.7356,-5.1341,2.5278,2.6721,4.8746$, $4.6415,2.0081,2.89,-4.1853,3.3412,-3.9702,1.3273,-1.8775,-0.3433$

To evaluate the performance of mismatched filter coefficients existing in Table 4, the ambiguity function was plotted as shown in Fig. 5 . 

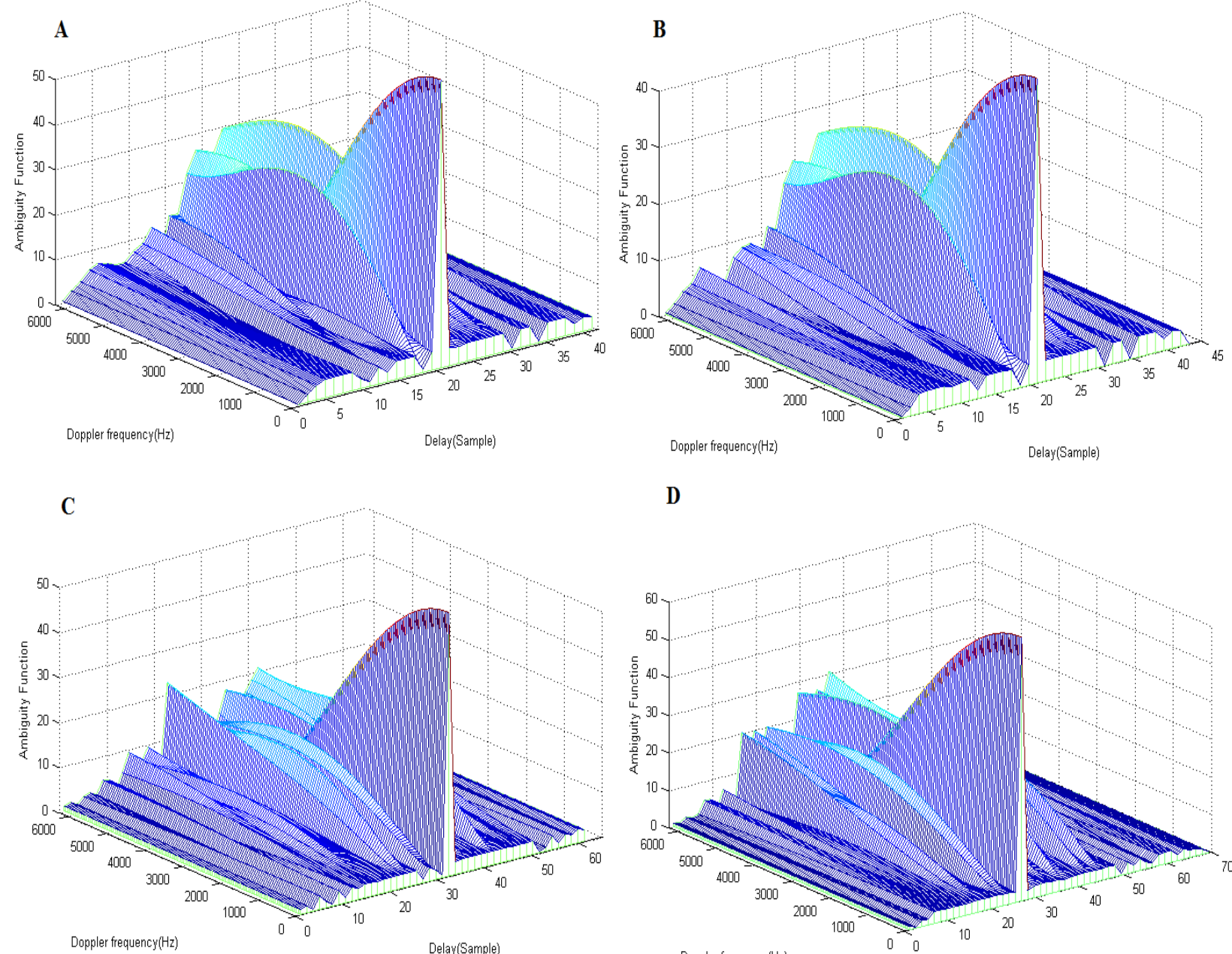

D

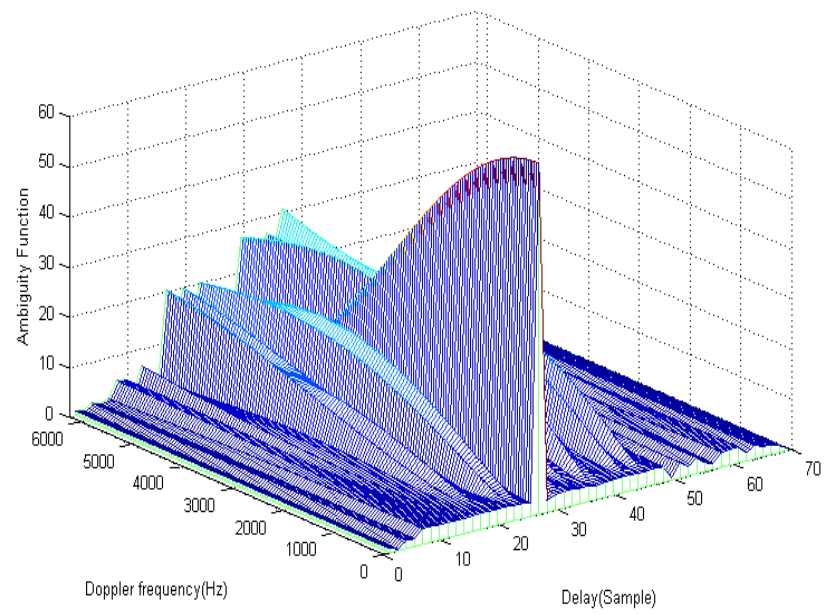

$\mathbf{E}$
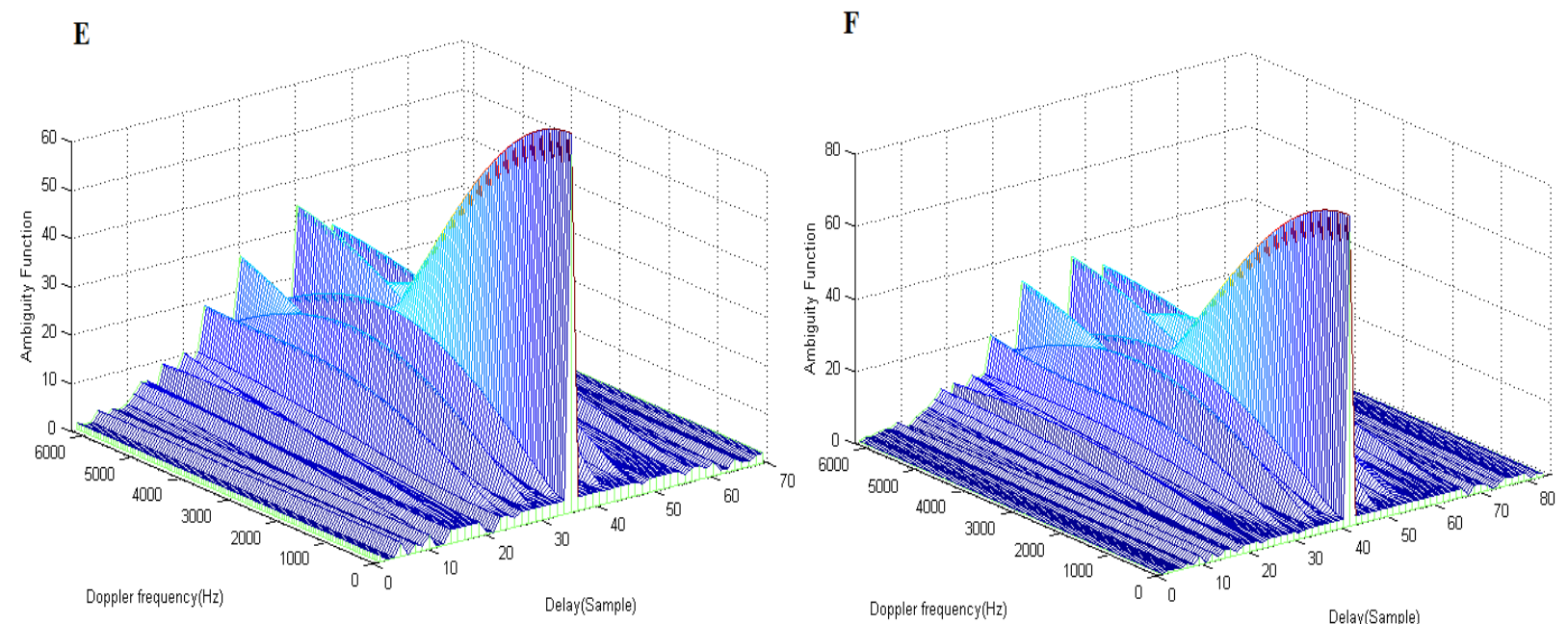

Fig.5. Ambiguity Function of the Mismatched Filter (A) 14/21 Cross-Correlation Function (B) 14/22 Cross-Correlation Function(C) 15/31 CrossCorrelation Function(D) 15/35 Cross-Correlation Function(E) 21/35 Cross-Correlation Function(F) 21/42 Cross-Correlation Function

\section{CONCLUSIONS}

In this paper, we considered the problem of high sidelobe levels in the output of the correlation function of matched filter. By using Genetic Algorithm, we found binary phase codes with minimum sidelobe levels which are presented in Table 2. To evaluate the performance of binary phase codes, the ambiguity function was plotted for these codes as shown in Fig. 3. To raise the peak to sidelobe rate of Mismatched Filter, we used Genetic 
Algorithms in order to generate filter coefficients. We could generate mismatched filter coefficients with different lengths and maximum PSR for binary phase codes. To test this, using the values mentioned in Table 3 , the ambiguity functions are plotted and shown in Fig. 5. Performance of this filter in presence of Doppler shift is acceptable.

\section{REFERENCES}

[1] Carroll J. Nunn, Gregory E. Coxson, "Best-Known Autocorrelation Peak Sidelobe Levels for Binary Codes of Length 71 to 105", International Workshop on Coding and Cryptography, April 15-19, 2013.

[2] Leukhin A.N., Potehin E.N, "Binary Sequences with Minimum Peak Sidelobe Level up to Length 68", International Workshop on Coding and Cryptography, April 15-19, 2013.

[3] A. NagaJyothi, Prof. K. Raja Rajeswari, "Crosscorrelation of Barker code and Long binary signals", International Journal of Engineering Science and Technology (IJEST), Vol. 3, 2011.

[4] Li, Lihua, Coon, Michael, McLinden, Matthew, "Radar Range Sidelobe Reduction Using Adaptive Pulse Compression Technique", NASA Tech Briefs, October, 2013.

[5] Maryam Amin Nasrabadi, Mohammad Hassan Bastani, "A Survey on the Design of Binary Pulse Compression Codes with Low Autocorrelation", Trends in Telecommunications Technologies, Christos J Bouras(Ed.) , 2010, ISBN: 978-953-307-072-8, InTech.

[6] Enrique Garcia, José A. Paredes, Fernando J. Álvarez, "Spreading sequences in active sensing", Signal Processing, Vol. 106 , pp. 88-105, 2015.

[7] G. L. Turin, "An Introduction to Matched Filters", IRE Trans, Vol. IT-6 pp. 311-329, 1960.

[8] Charles E. Cook and Howard S. Marsh, "An Introduction to Spread Spectrum" IEEE Communications Magazine, pp. 8-16, 1999.

[9] Anatolii N. Leukhin, Egor N. Potekhin, "Optimal Peak Sidelobe Level Sequences up to Length 74", Proceedings of the 43rd European Microwave Conference, , Nuremberg, Germany, 7-10 Oct, 2013.

[10] Matthew A. Ferrara, "Near-Optimal Peak Sidelobe Binary Codes" IEEE Department of Mathematical Sciences Rensselaer Polytechnic Institute Troy, New York 12180, pp. 400-403, 2006.

[11] Georges R. Harik, Fernando G. Lobo, and David E. Goldberg, "The Compact Genetic Algorithm", IEEE Transactions on Evolutionary Computation, Vol. 3, No. 4, 1999.
[12] Dept. of Electr. Eng., Idaho Univ., Moscow, ID, USA, "Genetic algorithms", Potentials IEEE, Vol. 12, No. 3 , pp. $21-24,1993$.

[13] Jie Chen, Ron J. Patton, "Robust Residual Generator Design via Multi-Objective Optimization and Genetic Algorithms", the International Series on Asian Studies in Computer and Information Science, Vol. 3, pp. 167-192, 1999.

[14] Wen-Yang Lin, Wen-Yuan Lee, and Tzung-Pri Hong, "Adapting Crossover and Mutation Rates in Genetic Algorithms", Journal of Information Science and Engineering, Vol. 19, pp. 889-903, 2003.

[15] Yilmaz KAYA, Murat UYAR, Ramazan TEKDN, "A Novel Crossover Operator for Genetic Algorithms: Ring Crossover", Computing Research Repository Journal, Vol. abs/1105.0, 2011.

[16] I. Abuiziah, N. Shakarneh, "A Review of Genetic Algorithm Optimization: Operations and Applications to Water Pipeline Systems", International Journal of Mathematical, Computational, Physical and Quantum Engineering, Vol. 7, No. 12, pp. 1262-1268, 2013.

[17] G. K. Mahanti and A. Chakrabarty, "Phase-Only and Amplitude-Phase Synthesis of Dual-Pattern Linear Antenna Arrats Using Floating-Point Genetic Algorithms", Progress In Electromagnetics Research, Vol. 68, pp. 247259, 2007.

\section{Authors' Profiles}

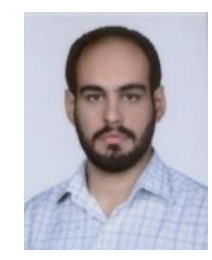

Hesam Ghaferi received his M.S. degree in Electrical Engineering from the Islamic Azad University, Sepidan, Iran, during 2012-2014 and Electronics Engineering degree from University of Applied Science and Technology ITMC, Shiraz, Iran. His research interests include Digital signal processing, optimization techniques and implementation of signal processing based on FPGA.

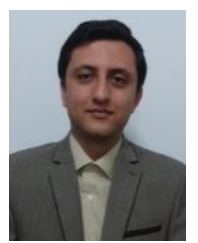

Mohammad Mehdi Pishrow obtained his M.S. degree in Communication Engineering at Shiraz University in 2011. He has specialized in beamforming and optimization techniques. His research interests always include statistical signal processing.

How to cite this paper: Hesam Ghaferi, Mohammad Mehdi Pishrow,"Optimization of Matched and Mismatched Filters in Short Range Pulse Radars using Genetic Algorithm", International Journal of Image, Graphics and Signal Processing(IJIGSP), Vol.8, No.5, pp.43-51, 2016.DOI: 10.5815/ijigsp.2016.05.03 